\title{
The behavior of renormalizations of circle maps with rational rotation numbers and with Zygmund conditions
}

\author{
Habibulla Akhadkulov a, ${ }^{*}$, Abdumajid Begmatov b, c, Teh Yuan Ying a \\ a School of Quantitative Sciences, Universiti Utara Malaysia, CAS 06010, UUM Sintok, Kedah Darul Aman, Malaysia. \\ ${ }^{b}$ Faculty of Mathematics, National University of Uzbekistan, University Street 4, Olmazor District, Tashkent 100174, Uzbekistan. \\ ${ }^{c}$ Department of Natural and Mathematical Sciences, Turin Polytechnic University in Tashkent, Kichik Halka yuli 17, Tashkent 100095, Uzbekistan \\ *Corresponding author: habibulla@uum.edu.my
}

\section{Article history}

Received 19 August 2019

Revised 30 January 2020

Accepted 20 April 2020

Published Online 17 August 2020

\begin{abstract}
Let $f_{t}=f_{0}+t(\bmod 1)$ be one-parameter family of circle homeomorphisms with a break point, that is, the derivative $D f_{0}$ has jump discontinuity at this point. Suppose $D f$ satisfies a certain Zygmund condition which is dependent on parameter $\gamma>0$. We prove that the renormalizations of circle homeomorphisms from this family with rational rotation number of sufficiently large rank are approximated by piecewise fractional linear transformations in $C^{1+L_{1}}$ and $C^{2}$-norms, depending on the values of the parameter $\gamma \in(1 / 2,1]$ and $\gamma \in(1,+\infty)$, respectively.
\end{abstract}

Keywords: Renormalization, rotation number, break point.

\section{INTRODUCTION}

One of the most studied classes of dynamical systems are orientation-preserving homeomorphisms of the circle $S^{1}=R^{1} / \mathrm{Z}^{1}$. Poincare (1885) noticed that the orbit structure of an orientationpreserving diffeomorphism $f$ is determined by some irrational mod 1, the rotation number $\rho=\rho(f)$ of $f$, in the following sense: for any $\xi \in S^{1}$ the mapping $f^{j}(\xi) \rightarrow j \rho(\bmod 1), j \in Z^{1}$ is orientation-preserving. Denjoy proved that if $f$ is an orientationpreserving $C^{1}$ - diffeomorphism of the circle with irrational rotation number $\rho$ and $\log f^{\prime}$ has bounded variation, then the orbit $\left\{f^{j}(\xi)\right\}_{j \in Z^{1}}$ is dense and the mapping $f^{j}(\xi) \rightarrow j \rho(\bmod 1)$ can therefore be extended by continuity to a homeomorphism $h$ of circle which conjugates $f$ to the linear rotation $f_{\rho}: \xi \rightarrow \xi+\rho(\bmod 1)$. In this context, it is a natural question to ask, under what conditions the conjugation is smooth. The first local results, that is the results requiring the closeness of diffeomorphism to the linear rotation, were obtained by Arnold [1] and Moser [17]. Next, Herman [10] obtained a first global result (i.e. not requiring the closeness of diffeomorphism to the linear rotation) asserting regularity of conjugation of the circle diffeomorphism. His result was developed by Yoccoz [19], Stark [18], Khanin and Sinai [13, 14], Katznelson and Ornstein [12], Khanin and
Teplinsky [15], Akhadkulov et al. [3]. They have shown that if $f$ is $C^{3}$ or $C^{2+\alpha}$ and rotation number $\rho$ satisfies certain Diophantine condition, then the conjugation will be at least $C^{1}$. Notice that the renormalization approach used in [14] and [18] is more natural in the spirit of Herman's theory. In this approach, regularity of the conjugation can be obtained by using the convergence of renormalizations of sufficiently smooth circle diffeomorphisms. In fact, the renormalizations of a smooth circle diffeomorphism converge exponentially fast to a family of linear maps with slope 1 . Such a convergence together with the condition on the rotation number (of Diophantine type) imply the regularity of conjugation. A natural generalization of diffeomorphisms of the circle are homeomorphisms with break points, i.e., those circle diffeomorphisms which are smooth everywhere with the exception of finitely many points at which their derivatives have jump discontinuities. Circle homeomorphisms with breaks were investigated by Herman [10] in the piecewise-linear (PL) case. The studies of more general (non PL) circle diffeomorphisms with a unique break point started with the work of Khanin and Vul [16]. Apparently, the renormalizations of circle homeomorphisms with break points are rather different from those of smooth diffeomorphisms. Indeed, the renormalizations of such a circle diffeomorphism converge exponentially fast to a two-parameter family of Möbius transformations. Applications of their result are very wide in many branches of one-dimensional dynamics, examples are the investigation of the invariant measures, nontrivial scaling and 
prevalence of periodic trajectories in one parameter families. In particular, they investigated the renormalization in the case of rational rotation number. Using convexity of the renormalization analyzed positions of periodic trajectories of one parameter family of circle maps and they proved that the rotation number is rational for almost all parameter values.

The purpose of the present work is to study the asymptotical behavior of the renormalizations of circle homeomorphisms with rational rotation number of enough large rank and satisfying a certain Zygmund condition dependent on a parameter $\gamma>0$. In Theorem 1, we show that if $\gamma \in(1 / 2,1]$, then the renormalizations converge to the piecewise Möbius transformations with speed $O\left(\mathrm{n}^{-\gamma}\right)$ in $C^{1}$ norm and the second derivative of the renormalizations converge to the piecewise Möbius transformations with speed $O\left(\mathrm{n}^{-(\gamma-1)}\right)$ in $L_{1}$ -norm. Moreover, we show that if $\gamma \in(1,+\infty)$, then the second derivative of the renormalization convergence to the piecewise Möbius transformations with speed $O\left(\mathrm{n}^{-(\gamma-1)}\right)$ in $C^{0}$-norm. Since the speed of convergence of renormalizations is shown in explicit form. we believe that this result will have many applications in the future.

\section{Renormalizations of circle maps with rational rotation numbers}

Consider one-parameter family of circle homeomorphisms $f_{t}(x), x \in S^{1}$ with a break point $x_{b}$, that is,

$f_{t}(x)=f_{0}(x)+t(\bmod 1), \quad \mathrm{x} \in \mathrm{S}^{1}, \quad t \in[0,1)$,

where $f_{0}$ satisfies the following conditions:

(i) $f_{0} \in C^{1}\left(\left[\mathrm{x}_{b}, \mathrm{x}_{b}+1\right]\right)$;

(ii) inf $f_{0}^{\prime}>0$;

(iii) $f_{0}$ has one-sided derivatives $f_{0}^{\prime}\left(\mathrm{x}_{b} \pm 0\right)>0$ and $\sigma=\sqrt{f_{0}^{\prime}\left(\mathrm{x}_{b}-0\right) / \mathrm{f}_{0}^{\prime}\left(\mathrm{x}_{b}+0\right)} \neq 1$.

The number $\sigma$ is called size of break. The rotation number $\rho_{t}=\rho\left(\mathrm{f}_{t}\right)$ is defined as follows:

$$
\rho_{t}=\lim _{n \rightarrow \infty} \frac{F_{t}^{n}(x)}{n}(\bmod 1), \mathrm{x} \in \mathrm{R}^{1}, \quad \text { where } F_{t}=\mathrm{f}_{0}+t .
$$

Fixing an arbitrary rational number $p / q \in(0,1)$, then it can be expressed as a finite continued fraction expansion: $p / q=\left[\mathrm{k}_{1}, \mathrm{k}_{2}, \ldots, \mathrm{k}_{n}\right], \mathrm{k}_{n}>1$. We refer to the number $r(p / q)=n$ as the rank of rational number $p / q$. To be emphasize that the rational number depends on its rank $n$, we set $p_{n}:=p, q_{n}:=q$. Let $p_{m} / q_{m}=\left[\mathrm{k}_{1}, \mathrm{k}_{2}, \ldots, \mathrm{k}_{m}\right], 1 \leq m \leq n$ be "rational convergence" for $p / q$. The denominators $q_{m}$ satisfy the recursive relations: $q_{m}=k_{m} q_{m-1}+q_{m-2}$ for $1 \leq m \leq n$, with initial conditions $\quad q_{0}=1, q_{-1}=0$. Now consider the circle homeomorphisms $f_{t}$ from the family (1), with rational rotation number $\quad \rho_{t}=p / q$. Let $I(p / q)=\left\{\mathrm{t}: \rho_{t}=\mathrm{p} / \mathrm{q}\right\}$. Fix $t \in I(p / q)$ and denote $f:=f_{t}$. Since the rotation number $\rho_{t}=p / q$ is rational, for each $t \in I(p / q)$ the map $f_{t}$ admits at least one periodic orbit with period $q:=q_{n}$. Let $O_{f}\left(\mathrm{r}_{0}, \mathrm{q}_{n}\right)=\left\{\mathrm{r}_{i}=\mathrm{f}^{i}\left(\mathrm{r}_{0}\right), \mathrm{i}=0,1, \ldots,\left(\mathrm{q}_{n}-1\right)\right\} \quad$ be $\quad$ an arbitrary periodic orbit with period $q_{n}$. Taking an arbitrary point on the orbit $x_{0} \in O_{f}\left(\mathrm{r}_{0}, \mathrm{q}_{n}\right)$, below we define the finite sequence of dynamical partitions $P_{1}, P_{2}, \ldots, P_{n}$ of the circle. Denoted by $\Delta_{0}^{(\mathrm{m})}$ the intervals $\left[\mathrm{x}_{q_{m}}, \mathrm{x}_{0}\right]$, for $m$ odd, and $\left[\mathrm{x}_{0}, \mathrm{x}_{q_{m-1}}\right]$, for $m$ even. The intervals $\Delta_{i}^{(\mathrm{m}-1)}=f^{i}\left(\Delta_{0}^{(m-1)}\right)$, for $i=0,1, \ldots, q_{m}$ and $\Delta_{j}^{(\mathrm{m})}=f^{j}\left(\Delta_{0}^{(m)}\right)$, for $j=0,1, \ldots, q_{m-1}$, cover the whole circle (without overlapping except at end points) and form the $m$ th dynamical partition $\left\{\mathrm{P}_{m}\right\}$, for $m=0,1, \ldots, n-1$. Consider the system of intervals: $\mathrm{P}_{n}=\left\{\Delta_{i}^{(\mathrm{n}-1)}, \mathrm{i}=0,1, \ldots,\left(\mathrm{q}_{n}-1\right)\right\} . \quad$ Endpoints of the intervals $\Delta_{i}^{(\mathrm{n}-1)}=\left[\mathrm{f}^{i}\left(\mathrm{x}_{0}\right), \mathrm{f}^{i}\left(\mathrm{x}_{q_{n-1}}\right)\right], \quad 0 \leq i \leq q_{n}-1$ are periodic points with period $q_{n}$ and the system of intervals $P_{n}$, cover the whole circle and it is called partition generated by periodic orbit $O_{f}\left(\mathrm{r}_{0}, \mathrm{q}_{n}\right)$. Denoted by $\left[\mathrm{y}_{1}, \mathrm{y}_{2}\right]$, the interval formed by consequent points of the orbit $O_{f}\left(\mathrm{r}_{0}, \mathrm{q}_{n}\right)$ which contains the break point $x_{b}$. Introduce normalized coordinates $z$ in the interval $\left[\mathrm{y}_{1}, \mathrm{y}_{2}\right]$ by the formula $\mathrm{x}=\mathrm{y}_{1}+\mathrm{z}\left(\mathrm{y}_{2}-\mathrm{y}_{1}\right)$. Now we define the function $f_{n}(z)$ corresponding to the return map $f^{q_{n}}(x)$ in the normalized coordinate system:

$$
f_{n}(z)=\frac{f^{q_{n}}\left(\mathrm{y}_{1}+\mathrm{z}\left(\mathrm{y}_{2}-\mathrm{y}_{1}\right)\right)-\mathrm{y}_{1}}{y_{2}-y_{1}}, \quad z \in[0,1]
$$

The map $f_{n}(z)$ is called renormalization of homeomorphism $f$ on the interval $\left[\mathrm{y}_{1}, \mathrm{y}_{2}\right]$. Notice that here $n$ is the rank of rational rotation number $\rho_{t}=p / q, \quad t \in I(\mathrm{p} / \mathrm{q})$.

\section{Zygmund class and main results}

In order to formulate our result, we have to define a new class of circle maps. For this, we consider the function $Z_{\gamma}:[0,1) \rightarrow(0,+\infty)$ such that $Z_{\gamma}(0)=0$ and $Z_{\gamma}(x)=(-\log x)^{-\gamma}, x \in(0,1), \gamma>0$. Let $f$ be the circle homeomorphism with a break point $x_{b}$. Without loss of generality we assume $x_{b}=0$. Denote by $\Delta^{2} f^{\prime}(\zeta, \tau)$ the second symmetric difference of $\mathrm{f}^{\prime}$ on $[0,1]$ i.e.,

$$
\Delta^{2} f^{\prime}(\zeta, \tau)=\mathrm{f}^{\prime}(\zeta+\tau)+\mathrm{f}^{\prime}(\zeta-\tau)-2 \mathrm{f}^{\prime}(\zeta)
$$

where $\zeta \in[0,1]$ and $\tau \in[0,1 / 2]$ such that $\zeta+\tau, \zeta-\tau \in[0,1]$. Suppose that there exists a constant $C>0$ such that the following inequality holds:

$$
\left\|\Delta^{2} f^{\prime}(\cdot, \tau)\right\|_{L^{\infty}([0,1])} \leq C \tau Z_{\gamma}(\tau)
$$

We remind that the class of real functions satisfying (2) with $Z_{\gamma}(\tau)=1$ is called the Zygmund class [20]. Hu and Sullivan [11] applied this class to the theory of circle homeomorphisms for the first time. Denoted by $H^{1+Z_{\gamma}}\left(\mathrm{S}^{1} \backslash\left\{\mathrm{x}_{b}\right\}\right)$, the class of circle homeomorphisms $f$ with a break point $x_{b}$, whose derivative $\mathrm{f}^{\prime}$ has bounded variation and satisfying conditions (i)-(iii), and the inequality (2). Piecewise Möbius transformation is defined as follows: 


$$
G_{d}(z)= \begin{cases}\frac{\sigma^{3} z}{\sigma^{2}(\sigma-1) \mathrm{z}+\mathrm{d}\left(\sigma^{2}-1\right)+1}, & \text { if } \quad z \in[0, \mathrm{~d}], \\ \frac{\sigma z+d \sigma\left(\sigma^{2}-1\right)}{(\sigma-1) \mathrm{z}+\mathrm{d} \sigma\left(\sigma^{2}-1\right)+1}, & \text { if } \quad z \in[\mathrm{d}, 1] .\end{cases}
$$

where $d=\left(\mathrm{x}_{b}-\mathrm{y}_{1}\right) /\left(\mathrm{y}_{2}-\mathrm{y}_{1}\right)$. Our main result is the following Theorem 1. Let $f_{t} \in H^{1+Z_{\gamma}}\left(\mathrm{S}^{1} \backslash\left\{\mathrm{x}_{b}\right\}\right), \gamma>0, t \in I\left(\mathrm{p}_{n} / \mathrm{q}_{n}\right)$ be circle homeomorphisms from the family (1) with rational rotation number $\rho_{t}=p / q$ of rank $n$. Then, there are constant $C>0$ and natural number $n_{0}=n_{0}(f)$ such that, for all $n \geq n_{0}$ the following inequalities hold:

$\left\|\mathrm{f}_{n}-\mathrm{G}_{d}\right\|_{C^{1}([0,1] \backslash\{\mathrm{d}\})} \leq \frac{C}{n^{\gamma}}, \quad\left\|\mathrm{f}_{n}^{\prime \prime}-G_{d}^{\prime \prime}\right\|_{L_{1}([0,1], \mathrm{d} \ell)} \leq \frac{C}{n^{\gamma-1}}$, when

$\left\|\mathrm{f}_{n}-\mathrm{G}_{d}\right\|_{\left.C^{1}([0,1] \backslash \mathrm{d}\}\right)} \leq \frac{C}{n^{\gamma}}, \quad\left\|\mathrm{f}_{n}^{\prime \prime}-G_{d}^{\prime \prime}\right\|_{\mathrm{C}^{0}([0,1])} \leq \frac{C}{n^{\gamma-1}}$, when

where the constant $C$ is not depend on $n, t$ and chosen periodic orbit.

In the next sections, we will give a sketch of proof of Theorem 1 . Note that the class $H^{1+Z_{\gamma}}\left(\mathrm{S}^{1} \backslash\left\{\mathrm{x}_{b}\right\}\right), \gamma>0$ is wider than $C^{2+\alpha}$, but our estimations are weaker than Khanin and Vul's [16]. The proofs of main theorems based on distortion estimates. Zygmund condition plays an important role in the estimation the distortion. This allows for a considerable simplification of the proof and essentially makes it easier. Note also that similar results are obtained in [8], [9] with weaker estimation.

Estimates for the ratio of $f^{q_{n}}$-distortions and comparing relative coordinates with möbius functions

The distortion of the interval $I$ with respect to the function $f$ is $R(\mathrm{I} ; \mathrm{f})=\frac{|f(I)|}{|I|}$. The distortion is multiplicative with respect to composition: for any two functions $f$ and $g$ we have $R(\mathrm{I} ; \mathrm{f} \circ g)=R(\mathrm{I} ; g) \cdot \mathrm{R}(\mathrm{g}(\mathrm{I}) ; \mathrm{f})$. For any $x \in[\mathrm{a}, \mathrm{b}]$ we can consider the distortions: $R_{a}(x)=R([\mathrm{a}, \mathrm{x}] ; \mathrm{f})$ and $R_{b}(x)=R([\mathrm{x}, \mathrm{b}] ; \mathrm{f})$.

Now, we define relative coordinates on the intervals of dynamical partition $P_{n}$ and the ratio of $f^{q_{n}}$ - distortions, i.e. distortions of intervals with respect to $f^{q_{n}}$. Then, we describe the ratio of $f^{q_{n}}$ distortions by initial relative coordinates and we provide estimates for this description and its derivatives. Consider the dynamical partition $P_{n}$ which is generated by periodic orbit $O_{f}\left(\mathrm{r}_{0}, \mathrm{q}_{n}\right)$. Let $\left[\mathrm{y}_{1}, \mathrm{y}_{2}\right]$ the interval of $P_{n}$ which contains the break point $x_{b}$. If the point lies on the periodic orbit, then two intervals of the partition $P_{n}$ cover the point $x_{b}$ with endpoints. In this case as $\left[\mathrm{y}_{1}, \mathrm{y}_{2}\right]$ we take such that $\mathrm{y}_{1}=x_{b}$. To simplify, we take $x_{0}=y_{1}$. By assumption $\Delta_{0}^{(\mathrm{n}-1)}=\left[\mathrm{y}_{1}, \mathrm{y}_{2}\right]$ and consequently $f^{q_{n}}\left(\Delta_{0}^{(\mathrm{n}-1)}\right)=\Delta_{0}^{(\mathrm{n}-1)}$. Notice that the renormalization map $f_{n}$ is represented as a composition $f_{n}(z)=F_{2}\left(F_{1}(z)\right)$ of two functions $F_{1}$ and $F_{2}$, corresponding to maps $f: \Delta_{0}^{(n-1)} \rightarrow \Delta_{1}^{(n-1)}$ and $\quad f^{q_{n}-1}: \Delta_{1}^{(n-1)} \rightarrow \Delta_{q_{n}}^{(n-1)}=\Delta_{0}^{(n-1)}$, respectively. Introduce the relative coordinates $\mathrm{Z}_{i}: \Delta_{i}^{(n-1)} \rightarrow \Delta_{i+1}^{(n-1)}$ for all $0 \leq i \leq q_{n}-1$, by the formula:

$$
\mathrm{z}_{i}=\frac{f^{i}(x)-f^{i}\left(\mathrm{y}_{1}\right)}{f^{i}\left(\mathrm{y}_{2}\right)-f^{i}\left(y_{1}\right)}, \quad x \in \Delta_{0}^{(n-1)} .
$$

To simplify notions, we denote:

$$
a_{i}:=f^{i}\left(\mathrm{y}_{1}\right), \quad b_{i}:=f^{i}\left(\mathrm{y}_{2}\right), \quad x_{i}:=f^{i}(\mathrm{x}) \in \Delta_{i}^{(n-1)}, \quad 0 \leq i \leq q_{n}-1
$$

Put

$\gamma \in[1 / 2,1)$

$$
m_{n}=\exp \left\{\sum_{i=1}^{q_{n}-1} \frac{f^{\prime}\left(\mathrm{b}_{i}\right)-\mathrm{f}^{\prime}\left(\mathrm{a}_{i}\right)}{2 f^{\prime}\left(\mathrm{b}_{i}\right)}\right\}
$$

Since $\quad x_{b} \in \Delta_{0}^{(n-1)}=\left[y_{1}, y_{2}\right], \quad$ we consider intervals $\gamma \nexists_{l}^{(1-1)}+, \Phi \measuredangle i \leq q_{n}-1$ which no contain the break point. Denote

$U_{n}(\mathrm{f}(\mathrm{x}))=\log \frac{R\left(\left[\mathrm{f}\left(y_{1}\right), \mathrm{f}(x)\right] ; \mathrm{f}^{q_{n}-1}\right)}{R\left(\left[\mathrm{f}(x), \mathrm{f}\left(y_{2}\right)\right] ; \mathrm{f}^{q_{n}-1}\right)}-\log m_{n}, \quad x \in \Delta_{0}^{(n-1)}$.

Since $f(x)=a_{1}+z_{1}\left(b_{1}-a_{1}\right)$, we set

$$
U_{n}\left(\mathrm{z}_{1}\right):=\mathrm{U}_{n}\left(a_{1}+\mathrm{z}_{1}\left(\mathrm{~b}_{1}-\mathrm{a}_{1}\right)\right) .
$$

In the following lemma, it is provided the estimation for $U_{n}\left(\mathrm{z}_{1}\right)$, which are proved analogously as in [2], Lemmas 5.1.-5.4.

Lemma 1. Let $f \in H^{1+Z_{\gamma}}\left(\mathrm{S}^{1} \backslash\left\{\mathrm{x}_{b}\right\}\right), t \in I(\mathrm{p} / \mathrm{q})$ be circle homeomorphisms from the family (1) with rational rotation number $\rho=p / q$ of rank $n$. Then there are constant $C>0$ and natural number $n_{0}=n_{0}(f)$ such that, for all $n \geq n_{0}$ the following inequality holds:

$\max _{z_{1} \in[0,1]}\left|\mathrm{U}_{n}\left(\mathrm{z}_{1}\right)\right| \leq \frac{C}{n^{\gamma}}, \quad \max _{\mathrm{z}_{1} \in[0,1]}\left|\mathrm{z}_{1}\left(1-\mathrm{z}_{1}\right) \frac{\mathrm{dU}_{n}\left(\mathrm{z}_{1}\right)}{d z_{1}}\right| \leq \frac{C}{n^{\gamma}}$, when $\gamma \in(0,+\infty)$,

$\max _{z_{1} \in[0,1]}\left|\frac{\mathrm{dU}_{n}\left(\mathrm{z}_{1}\right)}{d z_{1}}\right| \leq \frac{C}{n^{\gamma-1}}, \max _{z_{1} \in[0,1]}\left|\mathrm{z}_{1}\left(1-\mathrm{z}_{1}\right) \frac{\mathrm{d}^{2} \mathrm{U}_{n}\left(\mathrm{z}_{1}\right)}{d z_{1}^{2}}\right| \leq \frac{C}{n^{\gamma-1}}$, when $\gamma \in(1,+\infty)$.

Next define relative coordinates of points of the intervals $\Delta_{q_{n}}^{(n-1)}=\Delta_{0}^{(n-1)}$ by

$$
\hat{\mathrm{z}}_{q_{n}-1}=\frac{f^{q_{n-1}}(y)-y_{1}}{y_{2}-y_{1}}, \text { where } y=a_{1}+z_{1}\left(b_{1}-a_{1}\right) .
$$

We show that relative coordinates $\hat{\mathrm{z}}_{q_{n}-1}$ are approximated by Möbius transformations of $\mathrm{z}_{1}$ for sufficiently large $n$. To characterize these approximations more precisely, we define a Möbius transformation as follows:

$$
M_{N}(z)=\frac{z N}{1+z(\mathrm{~N}-1)} .
$$

Lemma 2. Let $f \in H^{1+Z_{\gamma}}\left(\mathrm{S}^{1} \backslash\left\{\mathrm{x}_{b}\right\}\right), t \in I(\mathrm{p} / \mathrm{q}), \gamma \in(0,+\infty)$ be circle homeomorphisms from the family (1) with rational rotation number $\rho=p / q$ of rank $n$. Then, there are constant $C>0$ and natural number $n_{0}=n_{0}(f)$ such that, for all $n \geq n_{0}$ the following inequalities hold: 


$$
\left\|\hat{\mathrm{z}}_{q_{n}-1}-\mathrm{M}_{m_{n}}\right\|_{C^{1}([0,1])} \leq \frac{C}{n^{\gamma}} .
$$

Proof. First, we will find the explicit form of $\mathrm{z}_{q_{n}-1}$. A not too hard calculation shows that

$$
\frac{1-\hat{z}_{q_{n}-1}}{\hat{z}_{q_{n}-1}} \cdot \frac{z_{1}}{1-z_{1}}=\frac{R\left(\left[\mathrm{f}(x), \mathrm{f}\left(y_{2}\right)\right] ; \mathrm{f}^{q_{n}-1}\right)}{R\left(\left[\mathrm{f}\left(y_{1}\right), \mathrm{f}(x)\right] ; \mathrm{f}^{q_{n}-1}\right)}
$$

On the other hand, relation (4) implies that

$$
\frac{R\left(\left[\mathrm{f}(x), \mathrm{f}\left(y_{2}\right)\right] ; \mathrm{f}^{q_{n}-1}\right)}{R\left(\left[\mathrm{f}\left(y_{1}\right), \mathrm{f}(x)\right] ; \mathrm{f}^{q_{n}-1}\right)}=\frac{1}{m_{n}} \cdot \exp \left\{-\mathrm{U}_{n}\left(\mathrm{z}_{1}\right)\right\} .
$$

Therefore, the last two relations give us

$$
\frac{1-\hat{z}_{q_{n}-1}}{\hat{z}_{q_{n}-1}} \cdot \frac{z_{1}}{1-z_{1}}=\frac{1}{m_{n}} \cdot \exp \left\{-\mathrm{U}_{n}\left(\mathrm{z}_{1}\right)\right\} .
$$

Solving last equality for $\hat{\mathbf{Z}}_{q_{n}-1}$ gives

$$
\hat{\mathrm{z}}_{q_{n}-1}\left(\mathrm{z}_{1}\right)=\frac{\mathrm{z}_{1} m_{n}}{\left(1-\mathrm{z}_{1}\right) \exp \left\{-\mathrm{U}_{n}\left(\mathrm{z}_{1}\right)\right\}+\mathrm{z}_{1} m_{n}} .
$$

By Lemma 1, we have $\exp \left\{-\mathrm{U}_{n}\left(\mathrm{z}_{1}\right)\right\}=1+O\left(\frac{1}{n^{\gamma}}\right)$. Then equality (5) implies that

$$
\max _{z_{1} \in[0,1]}\left|\hat{\mathrm{z}}_{q_{n}-1}\left(\mathrm{z}_{1}\right)-\mathrm{M}_{m_{n}}\left(\mathrm{z}_{1}\right)\right| \leq \frac{C}{n^{\gamma}} .
$$

for all $n \geq n_{0}$. By differentiating equality (5) we obtain

$$
\hat{\mathrm{z}}_{q_{n}-1}^{\prime}\left(\mathrm{z}_{1}\right)=\frac{\left(1+z_{1}\left(1-\mathrm{z}_{1}\right) \mathrm{U}_{n}^{\prime}\left(\mathrm{z}_{1}\right)\right) m_{n} \exp \left\{-\mathrm{U}_{n}\left(\mathrm{z}_{1}\right)\right\}}{\left(\left(1-\mathrm{z}_{1}\right) \exp \left\{-\mathrm{U}_{n}\left(\mathrm{z}_{1}\right)\right\}+\mathrm{z}_{1} m_{n}\right)^{2}} .
$$

By Lemma 1, we have $\exp \left\{-\mathrm{U}_{n}\left(\mathrm{z}_{1}\right)\right\}=1+O\left(\frac{1}{n^{\gamma}}\right)$ and $\left|z_{1}\left(1-z_{1}\right) \mathrm{U}_{n}^{\prime}\left(\mathrm{z}_{1}\right)\right|=O\left(\frac{1}{n^{\gamma}}\right)$. Then last equality implies that

$\max _{z_{1} \in[0,1]}\left|\hat{\mathrm{z}}_{q_{n}-1}^{\prime}\left(\mathrm{z}_{1}\right)-\mathrm{M}_{m_{n}}^{\prime}\left(\mathrm{z}_{1}\right)\right| \leq \frac{C}{n^{\gamma}}$.

for all $n \geq n_{0}$. Inequalities (6) and (7) imply the assertion of Lemma 2.

The following lemma shows that for sufficiently large $n$, the relative coordinates $\hat{\mathrm{Z}}_{q_{n}}$ are approximated by Möbius transformations in $C^{2}$-norm when $\gamma>1$.

Lemma 3. Let $f \in H^{1+Z_{\gamma}}\left(\mathrm{S}^{1} \backslash\left\{\mathrm{x}_{b}\right\}\right), t \in I(\mathrm{p} / \mathrm{q}), \gamma \in(1,+\infty)$ be circle homeomorphisms from the family (1) with rational rotation number $\rho=p / q$ of rank $n$. Then there are constant $C>0$ and natural number $n_{0}=n_{0}(f)$ such that, for all $n \geq n_{0}$ the following inequalities hold:

$$
\left\|\hat{\mathrm{z}}_{q_{n}-1}-\mathrm{M}_{m_{n}}\right\|_{C^{1}([0,1)} \leq \frac{C}{n^{\gamma}}, \quad\left\|\hat{\mathrm{z}}_{q_{n}-1}^{\prime \prime}-\mathrm{M}_{m_{n}}^{\prime \prime}\right\|_{C^{0}([0,1])} \leq \frac{C}{n^{\gamma-1}} .
$$

Proof. First inequality in (8) immediately follows from Lemma 2. To prove second inequality of lemma we find explicit form for $\hat{Z}_{q_{n}-1}^{\prime \prime}$ :

$$
\begin{aligned}
& \hat{\mathrm{z}}_{q_{n}-1}^{\prime \prime}=\frac{m_{n} \exp \left\{-\mathrm{U}_{n}\left(\mathrm{z}_{1}\right)\right\}\left(\mathrm{U}_{n}^{\prime}\left(\mathrm{z}_{1}\right)\left(2 \mathrm{z}_{1}-\mathrm{Z}_{1}\left(1-\mathrm{z}_{1}\right) \mathrm{U}_{n}^{\prime}\left(\mathrm{z}_{1}\right)\right)-\mathrm{z}_{1}\left(1-\mathrm{z}_{1}\right) \mathrm{U}_{n}^{\prime \prime}\left(\mathrm{z}_{1}\right)\right)}{\left(\left(1-\mathrm{Z}_{1}\right) \exp \left\{-\mathrm{U}_{n}\left(\mathrm{z}_{1}\right)\right\}+\mathrm{z}_{1} m_{n}\right)^{2}} \\
& -\frac{2 m_{n} \exp \left\{-\mathrm{U}_{n}\left(\mathrm{z}_{1}\right)\right\}\left(1-\mathrm{Z}_{1}\left(1-\mathrm{z}_{1}\right) \mathrm{U}_{n}^{\prime}\left(\mathrm{z}_{1}\right)\right)\left(m_{n}-\exp \left\{-\mathrm{U}_{n}\left(\mathrm{z}_{1}\right)\right\}+\left(1-\mathrm{Z}_{1}\right) \mathrm{U}_{n}^{\prime}\left(\mathrm{z}_{1}\right)\right)}{\left(\left(1-\mathrm{Z}_{1}\right) \exp \left\{-\mathrm{U}_{n}\left(\mathrm{z}_{1}\right)\right\}+\mathrm{z}_{1} m_{n}\right)^{3}} .
\end{aligned}
$$

This equality and Lemma 1 imply second inequality in (8). We are done.

It follows from the definition of relative coordinates of $z_{i}$ that the functions $F_{1}$ and $F_{2}$ can be written as follows:

$$
\begin{aligned}
F_{1}\left(\mathrm{z}_{0}\right) & =\frac{f\left(\mathrm{y}_{1}+z_{0}\left(\mathrm{y}_{2}-\mathrm{y}_{1}\right)\right)-\mathrm{f}\left(\mathrm{y}_{1}\right)}{f\left(\mathrm{y}_{2}\right)-\mathrm{f}\left(\mathrm{y}_{1}\right)} \\
F_{2}\left(\mathrm{z}_{1}\right) & =\frac{f^{q_{n}-1}\left(\mathrm{f}\left(\mathrm{y}_{1}\right)+z_{1}\left(\mathrm{f}\left(\mathrm{y}_{2}\right)-f\left(\mathrm{y}_{1}\right)\right)\right)-\mathrm{y}_{1}}{\mathrm{y}_{2}-\mathrm{y}_{1}}
\end{aligned}
$$

and $f_{n}(\mathrm{z})=F_{2}\left(\mathrm{~F}_{1}(\mathrm{z})\right)$. Define the following piecewise fractional linear function:

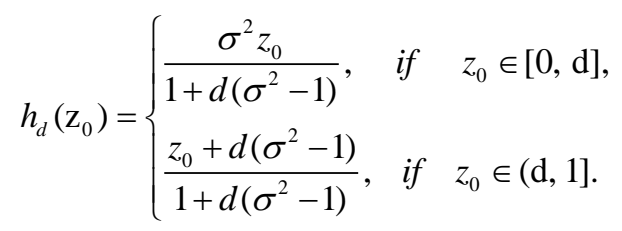

Lemma 4. Let $f \in H^{1+Z_{\gamma}}\left(\mathrm{S}^{1} \backslash\left\{\mathrm{x}_{b}\right\}\right), t \in I(\mathrm{p} / \mathrm{q}), \gamma \in(0,+\infty)$ be circle homeomorphisms from the family (1) with rational rotation number $\rho=p / q$ of rank $n$. Then there are constants $C>0$, $0<\lambda<1$ and natural number $n_{0}=n_{0}(f)$ such that, for all $n \geq n_{0}$ the following inequalities hold:

$$
\begin{gathered}
\left\|\mathrm{F}_{1}-h_{d}\right\|_{\left.C^{1}([0,1] \backslash \mathrm{d}\}\right)} \leq C \lambda^{n}, \quad \text { if } \quad \gamma \in(0,1 / 2] ; \\
\left\|\mathrm{F}_{1}-h_{d}\right\|_{\left.C^{2}([0,1] \backslash \mathrm{d}\}\right)} \leq C \lambda^{n}, \quad \text { if } \quad \gamma \in(1,+\infty) ; \\
\left\|\mathrm{F}_{1}-h_{d}\right\|_{C^{1}([0,1] \backslash\{\mathrm{d}\})} \leq C \lambda^{n}, \quad \mid \mathrm{F}_{1}^{\prime} \|_{\mathrm{L}_{1}([0,1], \mathrm{d} \ell)} \leq C \lambda^{n}, \quad \text { if } \quad \gamma \in(1 / 2,1] .
\end{gathered}
$$

Proof, Let $y_{1}<x<x_{b}$. Since $f^{\prime}$ satisfies the condition (2), we obtain

$$
\begin{aligned}
& \frac{f(\mathrm{x})-\mathrm{f}\left(\mathrm{y}_{1}\right)}{x-y_{1}}=\frac{1}{x-y_{1}} \int_{0}^{\frac{x-y_{1}}{2}}\left(f^{\prime}\left(\frac{x+y_{1}}{2}+y\right)+f^{\prime}\left(\frac{x+y_{1}}{2}-y\right)\right) d y= \\
& =\frac{1}{x-y_{1}} \int_{0}^{\frac{x-y_{1}}{2}}\left(2 f^{\prime}\left(\frac{x+y_{1}}{2}\right)+O\left(\left|\Delta_{0}^{(\mathrm{n}-1)}\right| \mathrm{Z}_{\gamma}\left(\left|\Delta_{0}^{(\mathrm{n}-1)}\right|\right)\right)\right) d y \\
& =\frac{f^{\prime}(\mathrm{x})+\mathrm{f}^{\prime}\left(\mathrm{y}_{1}\right)}{2}+O\left(\left|\Delta_{0}\right| \mathrm{Z}_{\gamma}\left(\left|\Delta_{0}\right|\right)\right) .
\end{aligned}
$$

Then we get

$$
\begin{aligned}
& f(x)-f\left(y_{1}\right)=\left(\mathrm{x}-\mathrm{y}_{1}\right) \frac{f^{\prime}(x)+f^{\prime}\left(\mathrm{y}_{1}\right)}{2} \\
& +\left(\mathrm{x}-\mathrm{y}_{1}\right) \mathrm{O}\left(\left|\Delta_{0}\right| \mathrm{Z}_{\gamma}\left(\left|\Delta_{0}\right|\right)\right), \quad \mathrm{y}_{1}<x<x_{b} .
\end{aligned}
$$

Similar calculations show that 


$$
\begin{aligned}
& f(x)-f\left(\mathrm{x}_{b}\right)=\left(\mathrm{x}-x_{b}\right) \frac{f^{\prime}(x)+f^{\prime}\left(\mathrm{x}_{b}+0\right)}{2} \\
& +\left(\mathrm{x}-x_{b}\right) \mathrm{O}\left(\left|\Delta_{0}\right| \mathrm{Z}_{\gamma}\left(\left|\Delta_{0}\right|\right)\right), \quad x_{b}<x<y_{2}, \\
& \quad f\left(\mathrm{y}_{2}\right)-f\left(\mathrm{x}_{b}\right)=\left(\mathrm{y}_{2}-x_{b}\right) \frac{f^{\prime}\left(x_{b}+0\right)+f^{\prime}\left(\mathrm{y}_{2}\right)}{2} \\
& +\left(\mathrm{y}_{2}-x_{b}\right) \mathrm{O}\left(\left|\Delta_{0}\right| \mathrm{Z}_{\gamma}\left(\left|\Delta_{0}\right|\right)\right) . \\
& \quad f\left(\mathrm{x}_{b}\right)-f\left(\mathrm{y}_{1}\right)=\left(\mathrm{x}_{b}-y_{1}\right) \frac{f^{\prime}\left(\mathrm{y}_{1}\right)+f^{\prime}\left(\mathrm{x}_{b}-0\right)}{2} \\
& +\left(\mathrm{x}_{b}-y_{1}\right) \mathrm{O}\left(\left|\Delta_{0}\right| \mathrm{Z}_{\gamma}\left(\left|\Delta_{0}\right|\right)\right),
\end{aligned}
$$

It follows from Theorems 4.1 and 4.3 in [2] that

$\left|\mathrm{f}^{\prime}(\mathrm{x})-\mathrm{f}^{\prime}(\mathrm{y})\right| \leq \mathrm{C} \Omega(|\mathrm{I}| ; \gamma), \quad \mathrm{x}, \mathrm{y} \in \mathrm{I}$,

where

$$
\Omega(\delta ; \gamma)=\left\{\begin{array}{l}
\delta\left(\log \frac{1}{\delta}\right)^{1-\gamma}, \text { if }(\delta, \gamma) \in(0,1) \times(0,1), \\
\delta\left(\log \log \frac{1}{\delta}\right), \text { if }(\delta, \gamma) \in(0,1) \times\{1\}, \\
\delta, \text { if }(\delta, \gamma) \in(0,1) \times(1,+\infty) .
\end{array}\right.
$$

In fact, the function $\Omega(\delta ; \gamma)$ is the modulus of continuity of the functions satisfying relation (2) for the different cases of $\gamma$. Using the bound on continuity intervals of $f^{\prime}$, we get $f^{\prime}(x)=f^{\prime}\left(x_{b}-0\right)+O\left(\Omega\left(\left|\Delta_{0}\right| ; \gamma\right)\right), \quad y_{1}<x<x_{b} ; \quad f^{\prime}(x)=f^{\prime}\left(x_{b}+0\right)+O\left(\Omega\left(\left|\Delta_{0}\right| ; \gamma\right)\right), \quad x_{b}<x<y_{2} ;$

$$
\begin{aligned}
& f^{\prime}\left(\mathrm{y}_{1}\right)=f^{\prime}\left(\mathrm{x}_{b}-0\right)+\mathrm{O}\left(\Omega\left(\left|\Delta_{0}\right| ; \gamma\right)\right) \\
& f^{\prime}\left(\mathrm{y}_{2}\right)=f^{\prime}\left(\mathrm{x}_{b}+0\right)+\mathrm{O}\left(\Omega\left(\left|\Delta_{0}\right| ; \gamma\right)\right) .
\end{aligned}
$$

It is well known that $\left|\Delta_{0}\right| \leq C \lambda_{0}^{n}$ where $\lambda_{0}=\left(1+e^{-v}\right)^{-1 / 2}$ and $v$ is total variation of $\log f^{\prime}$ (see [3], [8]). It is easy to see that there exists $\lambda \in\left(\lambda_{0}, 1\right)$ such $\quad$ that $\left|\Delta_{0}\right| \mathrm{Z}_{\gamma}\left(\left|\Delta_{0}\right|\right)+\Omega\left(\left|\Delta_{0}\right| ; \gamma\right)=\mathrm{O}\left(\lambda^{n}\right)$. Using this we can rewrite relations (9)-(12) as:

$f(x)-f\left(y_{1}\right)=\left(\mathrm{x}-\mathrm{y}_{1}\right) \mathrm{f}^{\prime}\left(\mathrm{x}_{b}-0\right)+\left(\mathrm{x}-\mathrm{y}_{1}\right) \mathrm{O}\left(\lambda^{n}\right), \quad \mathrm{y}_{1}<x<x_{b}$.

$f(x)-f\left(x_{b}\right)=\left(x-x_{b}\right) f^{\prime}\left(x_{b}+0\right)+\left(x-x_{b}\right) O\left(\lambda^{n}\right), \quad x_{b}<x<y_{2}$,

$f\left(\mathrm{y}_{2}\right)-f\left(\mathrm{x}_{b}\right)=\left(\mathrm{y}_{2}-x_{b}\right) f^{\prime}\left(x_{b}+0\right)+\left(\mathrm{y}_{2}-\mathrm{x}_{b}\right) O\left(\lambda^{n}\right)$.

$f\left(x_{b}\right)-f\left(y_{1}\right)=\left(x_{b}-y_{1}\right) f^{\prime}\left(x_{b}-0\right)+\left(x_{b}-y_{1}\right) O\left(\lambda^{n}\right)$,

$$
\begin{aligned}
& F_{1}\left(\mathrm{z}_{0}\right)=\left\{\begin{array}{lll}
\frac{f(x)-f\left(y_{1}\right)}{f\left(\mathrm{y}_{2}\right)-f\left(x_{b}\right)+f\left(x_{b}\right)-f\left(y_{1}\right)}, & \text { if } \quad y_{1}<x<x_{b}, \\
\frac{f(x)-f\left(x_{b}\right)+f\left(x_{b}\right)-f\left(y_{1}\right)}{f\left(\mathrm{y}_{2}\right)-f\left(x_{b}\right)+f\left(x_{b}\right)-f\left(y_{1}\right)}, & \text { if } \quad & x_{b}<x<y_{2},
\end{array}=\right.
\end{aligned}
$$

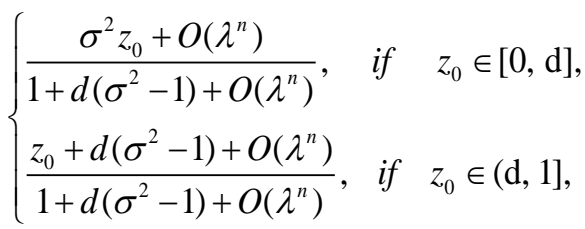

where $d=\left(\mathrm{x}_{b}-\mathrm{y}_{1}\right) /\left(\mathrm{y}_{2}-\mathrm{y}_{1}\right)$. Differentiating the initial form of $F_{1}\left(\mathrm{z}_{0}\right)$ and using relations (14)-(17), we have

$$
F_{1}^{\prime}\left(\mathrm{z}_{0}\right)=\frac{\left(\mathrm{y}_{2}-\mathrm{y}_{1}\right) \mathrm{f}^{\prime}(\mathrm{x})}{f\left(\mathrm{y}_{2}\right)-\mathrm{f}\left(\mathrm{y}_{1}\right)}=
$$

$$
\left\{\begin{array}{l}
\frac{\sigma^{2}+O\left(\lambda^{n}\right)}{1+d\left(\sigma^{2}-1\right)+O\left(\lambda^{n}\right)}, \quad \text { if } \quad z_{0} \in[0, \mathrm{~d}], \\
\frac{1+O\left(\lambda^{n}\right)}{1+d\left(\sigma^{2}-1\right)+O\left(\lambda^{n}\right)}, \quad \text { if } \quad z_{0} \in(\mathrm{d}, 1] .
\end{array}\right.
$$

So, we have proven that if $\gamma \in(0,+\infty)$, then the function $F_{1}\left(\mathrm{z}_{0}\right)$ is approximated by $h_{d}\left(\mathrm{z}_{0}\right)$ in $C^{1}$ - norm. Suppose that $\gamma>1$. According to Theorem 4.3 in [2], we have that $f \in C^{2}$. Then it is easy to see that

$$
\left|F^{\prime \prime}{ }_{1}\left(z_{0}\right)\right|=\frac{\left(\mathrm{y}_{2}-\mathrm{y}_{1}\right)^{2}\left|\mathrm{f}^{\prime \prime}(\mathrm{x})\right|}{\left|\mathrm{f}\left(\mathrm{y}_{2}\right)-\mathrm{f}\left(\mathrm{y}_{1}\right)\right|}=\frac{\left(\mathrm{y}_{2}-\mathrm{y}_{1}\right)\left|\mathrm{f}^{\prime \prime}(\mathrm{x})\right|}{\left|\mathrm{f}^{\prime}\left(\xi_{0}\right)\right|} \leq C \lambda^{n}
$$

where $\xi_{0} \in\left(\mathrm{y}_{1}, \mathrm{y}_{2}\right)$ and $\xi_{0} \neq x_{b}$. Similarly, one can show that $\left\|F^{\prime \prime}{ }_{1}\left(z_{0}\right)\right\|_{L_{1}} \leq C \lambda^{n}$, when $\gamma \in(1 / 2,1]$. This finishes the proof of Lemma 4.

\section{PROOF OF MAIN THEOREM}

To complete the proof of main theorem, we have to compare $m_{n}$ with $\sigma$.

Lemma 5. Let $f \in H^{1+Z_{\gamma}}\left(\mathrm{S}^{1} \backslash\left\{\mathrm{x}_{b}\right\}\right), t \in I(\mathrm{p} / \mathrm{q}), \gamma>1 / 2$ be circle homeomorphisms from the family (1) with rational rotation number $\rho=p / q$ of rank $n$. Then there are constant $C>0$, $0<\lambda<1$ and natural number $n_{0}=n_{0}(f)$ such that, for all $n \geq n_{0}$ the following inequality holds: $\left|\mathrm{m}_{n}-\sigma\right| \leq \mathrm{C} \lambda^{n}$.

(15) Proof. Since $\gamma>1 / 2$, it is easy to check that the following equalities hold: 


$$
\frac{f^{\prime}\left(\mathrm{b}_{i}\right)-f^{\prime}\left(\mathrm{a}_{i}\right)}{2 f^{\prime}\left(\mathrm{b}_{i}\right)}=\int_{a_{1}}^{b_{i}} \frac{f^{\prime \prime}(\mathrm{y})}{2 f^{\prime}(y)} d y+\int_{a_{1}}^{b_{i}} \frac{f^{\prime \prime}(\mathrm{y})}{2 f^{\prime}(y)}\left(\frac{f^{\prime}\left(\mathrm{x}_{i}\right)-f^{\prime}\left(\mathrm{a}_{i}\right)}{2 f^{\prime}\left(\mathrm{b}_{i}\right)}\right) d y .
$$

Since $\mid\left[\mathrm{a}_{i}, \mathrm{~b}_{i}\right] \leq \mathrm{C} \lambda^{n}, \quad i=0,1, \ldots,\left(\mathrm{q}_{n}-1\right)$, we have

$$
\int_{a_{1}}^{b_{i}} \frac{f^{\prime \prime}(\mathrm{y})}{2 f^{\prime}(y)}\left(\frac{f^{\prime}\left(\mathrm{x}_{i}\right)-f^{\prime}\left(\mathrm{a}_{i}\right)}{2 f^{\prime}\left(\mathrm{b}_{i}\right)}\right) d y=O\left(\lambda^{n} \int_{a_{i}}^{b_{i}}\left|\frac{f^{\prime \prime}(y)}{2 f^{\prime}(y)}\right| d y\right) .
$$

A not hard calculates show that

$$
\begin{aligned}
& \log m_{n}=\sum_{i=1}^{a_{n}-1} \frac{f^{\prime}\left(\mathrm{b}_{i}\right)-f^{\prime}\left(\mathrm{a}_{i}\right)}{2 f^{\prime}\left(\mathrm{b}_{i}\right)}=\sum_{i=1}^{a_{n}-1} \int_{a_{1}}^{b_{i}} \frac{f^{\prime \prime}(\mathrm{y})}{2 f^{\prime}(y)} d y-\int_{\Delta_{0}^{(\mathrm{n}-1)}} \frac{f^{\prime \prime}(\mathrm{y})}{2 f^{\prime}(y)} d y+O \\
& \text { Hence, according to the inequality } \int_{\Delta_{0}^{(\mathrm{n}-1)}}\left|\frac{f^{\prime \prime}(\mathrm{y})}{2 f^{\prime}(y)}\right| d y \leq C \lambda^{n} \text {, we get }
\end{aligned}
$$

the proof of Lemma 5. From the definitions of $F_{2}\left(\mathrm{z}_{1}\right)$ and $\hat{z}_{q_{n}-1}\left(\mathrm{z}_{1}\right)$

imply that $F_{2}\left(\mathrm{z}_{1}\right)=\hat{z}_{q_{n}-1}\left(\mathrm{z}_{1}\right)$. Then Lemmas 3 and 5 imply that

$$
\left\|\mathrm{F}_{2}-\mathrm{M}_{\sigma}\right\|_{C^{1}([0,1])} \leq \frac{C}{n^{\gamma}}, \quad\left\|\mathrm{F}_{2}^{\prime \prime}-\mathrm{M}_{\sigma}^{\prime \prime}\right\|_{C^{0}([0,1])} \leq \frac{C}{n^{\gamma-1}} .
$$

Hence, according to the relation $f_{n}(\mathrm{z})=F_{2}\left(\mathrm{~F}_{1}(\mathrm{z})\right)$ and Lemma 4 , we obtain the proof of Theorem 1 .

Remark. The Zygmund class satisfying inequality (2) is applied to circle homeomorphisms with irrational rotation numbers in [4]-[8]. However, to the best knowledge of authors, this is the first result where Zygmund smoothness condition is applied to circle homeomorphisms with rotational rotation number. We believe that in case of algebraic irrational rotation number, Theorem 1 can be applied in finding fixed points of the renormalization operator $R: f_{n} \rightarrow f_{n+1}$

\section{ACKNOWLEDGEMENT}

The authors are grateful to the editor-in-chief and referees for their accurate reading and useful suggestions. We would like to thank the Ministry of Education of Malaysia for providing us with the Fundamental Research Grant Scheme FRGS/1/2018/STG06/ UUM/02/13. Code S/O 14192.)

\section{REFERENCES}

[1] V. I. Arnol'd, Small denominators: I. Mappings from the circle onto itself. Izv. Akad. Nauk SSSR, Ser. Mat., 25, 21-86, (1961).

[2] H. Akhadkulov, M. S. Md Noorani, S. Akhatkulov, Renormalizations of circle diffeomorphisms with a break-type singularity. Nonlinearity, 30(7), 2687--2717, (2017).

[3] H. Akhadkulov, A. Dzhalilov, K. Khanin, Notes on a theorem of Katznelson and Ornstein. Discrete Contin. Dyn. Syst., 37(9), $4587-$ 4609, (2017).

[4] H. Akhadkulov, A. A. Dzhalilov, K. M. Khanin, On linearization of circle diffeomorphisms. Differential Equations and Dynamical Systems. Springer Proceeding in Mathematics \& Statistics, Springer, vol 268, pp.

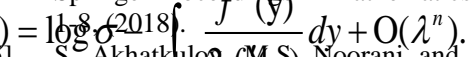

S. Akhatkulog, fM. $y$ ) Noorani and H. Akhadkulov, on the invarian measure $\Delta_{\emptyset \mathrm{f}}^{(\mathrm{n}-1)}$ a piecewise-smooth circle homeomorphism of Zygmund class. Journal of Nonlinear Sciences and Applications, 10(1), p. 48-59, (2017).

[6] H. Akhadkulov, M. S. Noorani, A. Saaban, S. Akhatkulov, Notes on Zygmund functions. International Journal of Pure and Applied Mathematics, 112(3), p 1-9, (2017).

[7] F. Alsharari, H. Akhadkulov, S. Akhatkulov, Remarks on "smooth" functions, International Journal of Mathematical Analysis, 10(25), p.1221-1228, (2016).

[8] A. Begmatov, Behavior of renormalizations of circle maps with rational rotation number, Uzbek Math. J., 2, 40-50, (2016).

[9] A. S. Begmatov, A. A. Dzhalilov, D. Mayer: Renormalizations of circle homeomorphisms with a single break point. Discrete Contin. Dyn. Syst., 34(11), 4487- 4513, (2014).

[10] M. Herman, Sur la conjugaison diffrentiable des diffomorphismes du cercle a des rotations. Publ. Math. Inst. Hautes, Etudes Sci., 49, 5-233, (1979).

[11] J. Hu, D. Sullivan, Topological conjugacy of circle diffeomorphisms. Ergod. Theor. Dyn. Syst, 17(1), 173-186, (1997).

[12] Y. Katznelson, D. Ornstein: The differentiability of the conjugation of certain diffeomorphisms of the circle. Ergod. Theor. Dyn. Syst., 9, 643680, (1989).

[13] K. Khanin, Ya. Sinai, A New Proof of M. Herman's Theorem. Comm. Math. Phys., 112, 89-101, (1987).

[14] K. Khanin, Ya. Sinai, Smoothness of conjugacies of diffeomorphisms of the circle with rotations. Russian Math. Surveys. 44, 69-99, (1989), translation of Uspekhi Mat. Nauk, 44, 57-82, (1989).

[15] K. Khanin, A. Teplinsky, Herman's theory revisited, Invent. Math., 178 (2), 333-344, (2009).

[16] K. Khanin, E. Vul, Circle homeomorphisms with weak discontinuities. In proc. of Dynamical systems and statistical mechanics (Moscow, 1991), 57-98. Amer. Math. Soc, Providence, RI, (1991).

[17] J. Moser, A rapid convergent iteration method and non-linear differential equations. II. Ann. Sc. Norm. Super. Pisa Cl. Sci., 20(3), 499-535, (1966).

[18] J. Stark, Smooth coniugacy and renormalisation for diffeomorphisms of the circle. Nonlinearity, 1, 541-575, (1988).

[19] J.-C. Yoccoz, Conjugaison differentiable des diffeomorphismes du cercle dont le nombre de rotation verifie une condition diophantienne. Ann. Sci. Ec. Norm. Super., 17, 333-361, (1984).

[20] A. Zygmund, Trigonometric series. Third Edition vol. I and II combined. Cambridge University Press. London (2002). 